YITP-96-55

hep-th/9610187

October 1996

\title{
Two-toroidal Lie Algebra as Current Algebra of Four-dimensional Kähler WZW Model
}

\author{
Takeo Inami, Hiroaki Kanno*, Tatsuya Ueno \\ and \\ Chuan-Sheng Xiong \\ Yukawa Institute for Theoretical Physics \\ Kyoto University, Kyoto 606-01, Japan \\ and \\ Department of Mathematics, Faculty of Science* \\ Hiroshima University, Higashi-Hiroshima 739, Japan
}

\begin{abstract}
We investigate the structure of an infinite-dimensional symmetry of the four-dimensional Kähler WZW model, which is a possible extension of the two-dimensional WZW model. We consider the $S L(2, R)$ group and, using the Gauss decomposition method, we derive a current algebra identified with a two-toroidal Lie algebra, a generalization of the affine Kac-Moody algebra. We also give an expression of the energy-momentum tensor in terms of currents and extra terms.
\end{abstract}




\section{Introduction}

Many important physical phenomena in particle physics, such as chiral symmetry breaking and confinement, are of a non-perturbative nature, and it is difficult to solve quantum field theories (QFT's) describing these phenomena. There is, however, a large class of 1+1D QFT's which share many of the important properties of 3+1D QFT's. Some such theories, e.g. the Gross-Neveu model and the sine-Gordon model, have been proven to be exactly solvable, and they have provided us with insight into the properties of their $3+1 \mathrm{D}$ counterparts.

The exact solvability of $1+1 \mathrm{D}$ QFT relies on the existence of an infinite number of conservation laws associated with infinite-dimensional symmetry, e.g. an affine KacMoody (KM) algebra symmetry in the Wess-Zumino-Witten (WZW) model. Continual attempts have been made toward constructing integrable QFT's in space-times of more than two dimensions [1], but without much progress. 4D Self-dual Yang-Mills and selfdual gravity equations have been shown to be completely integrable. However, these theories are at the classical level, and in four dimensions, no integrable QFT's based on Lagrangian have been constructed.

Recently a non-linear sigma model (NLSM) in four dimensions with a Wess-Zumino like term has been proposed as a possible extension of the 2D WZW model [2], [3]. It was first introduced by Donaldson [4] (see also [5]). In [2], this model, which we refer to as 4D Kähler WZW (KWZW) model, was studied in relation to the 5D Kähler Chern-Simons theory and was shown to have an infinite-dimensional symmetry. Later, it was shown that the model is solvable in some algebraic sector [3] and that it is one-loop on-shell finite [3], [6]. Interestingly, the KWZW model appears as an effective field theory of the $N=2$ string [7] and also as a chiral sector of the QCD scattering theory [8].

In this paper, we investigate the structure of the infinite-dimensional symmetry of the KWZW model in 4D flat space-time. To construct the current algebra for the symmetry explicitly and to simplify the argument, we consider the group $S L(2, R)$. The extension to other Lie groups will be mentioned later. By using the method of the Gauss decom- 
position, we derive the current algebra and identify it with a two-toroidal Lie algebra, which is a generalization of the affine KM algebra. We also give an expression for the energy-momentum tensor in terms of currents and extra terms, in the spirit of Sugawara's conjecture.

\section{The KWZW model and Gauss decomposition}

The basic field in the KWZW model is a mapping $g(x)$ from a four-manifold $X_{4}$ to a Lie group $G$. The action for $g(x)$ is given as a generalization of the 2D WZW model [2], [3],

$$
S=-\frac{i}{4 \pi} \int_{X_{4}} \omega \wedge \operatorname{Tr}\left(g^{-1} \partial g \wedge g^{-1} \bar{\partial} g\right)+\frac{i}{12 \pi} \int_{X_{5}} \omega \wedge \operatorname{Tr}\left(g^{-1} d g\right)^{3}
$$

The two-form $\omega$ is a closed Kähler form on $X_{4}$,

$$
\omega=\frac{i}{2} f_{\pi}^{2} h_{\alpha \bar{\beta}} d z^{\alpha} \wedge d \bar{z}^{\beta}
$$

where $h_{\alpha \bar{\beta}}(\alpha, \bar{\beta}=1,2)$ is a Kähler metric on $X_{4}$ and $f_{\pi}$ is a coupling constant with the mass dimension +1 . The equation of motion reads,

$$
\bar{\partial}\left(\omega \wedge g^{-1} \partial g\right)=0
$$

or equivalently,

$$
\partial\left(\omega \wedge \bar{\partial} g g^{-1}\right)=0
$$

These equations are known as the Yang equations on a Kähler manifold $X_{4}$ and are equivalent to the self-dual Yang-Mills (SDYM) equation in a particular gauge [9]. When we restrict $X_{4}$ to a hyper-Kähler manifold, the two-form $\omega$ represents a self-dual gravitational (SDG) instanton, since in four dimensions the hyper-Kähler condition is equivalent to the self-duality of the Riemann tensor. Then (3) or (4) describes a SDYM instanton coupled to a SDG instanton.

A comment is in order on the signature of the space-time $X_{4}$. The $(4,0)$ signature is favoured from the viewpoint of the SDYM equation, whereas the KWZW model arising 
from the $N=2$ string and that in the QCD prefer $(2,2)$ and $(3,1)$, respectively. In this paper, we will not be concerned with the signature of $X_{4}$, assuming that any two signatures are connected to each other by analytic continuation.

The integrable self-dual equation suggests the existence of an infinite-dimensional symmetry in the action (11). In fact, we can prove the following identity [2], [3], which is an analogue of the Polyakov-Wiegmann formula,

$$
S[g h]=S[g]+S[h]-\frac{i}{2 \pi} \int_{X_{4}} \omega \wedge \operatorname{Tr} g^{-1} \partial g \bar{\partial} h h^{-1}
$$

From this formula, we can easily see that the action is invariant under holomorphic right and anti-holomorphic left symmetries, $g \rightarrow h_{L}\left(\bar{z}^{1}, \bar{z}^{2}\right) g h_{R}\left(z^{1}, z^{2}\right)$. Corresponding to the right (left) action symmetry, we have a conserved current $J(\bar{J})$,

$$
J=\frac{i}{\pi} \omega \wedge g^{-1} \partial g, \quad \bar{J}=-\frac{i}{\pi} \omega \wedge \bar{\partial} g g^{-1}
$$

It was known that the SDYM equation has another type of symmetry associated with an infinite number of non-local conserved currents [10], which is different from the symmetry of the KWZW model associated with (6). The former symmetry leads to an affine KM algebra with no central extension. On the other hand, as will see below, we do have a central extension in the infinite-dimensional algebra for the KWZW model. The symmetry in [10] is an analogue of that in the 2D principal chiral model, whereas the symmetry in this paper is an analogue of that in the 2D WZW model.

Although the KWZW model is apparently non-renormalizable by power counting, it has been shown that the model is one-loop on-shell finite [3]. Conversely, the requirement of the one-loop finiteness of the NLSM with torsion leads to the KWZW model uniquely [6]. This remarkable property arises from the existence of torsion in group manifolds, represented by the Wess-Zumino like term, the second term in (11).

We consider the KWZW model with the $S L(2, R)$ group and use the Gauss decom- 
position method to express $g(x)$ in the neighborhood of the unit element as follows,

$$
g(x)=e^{\chi(x) E_{+}} e^{\varphi(x) H} e^{\psi(x) E_{-}}=\left[\begin{array}{cc}
e^{\varphi}+\chi \psi e^{-\varphi} & \chi e^{-\varphi} \\
\psi e^{-\varphi} & e^{-\varphi}
\end{array}\right],
$$

where $\varphi(x), \chi(x)$ and $\psi(x)$ are real scalar fields and

$$
H=\left[\begin{array}{cc}
1 & 0 \\
0 & -1
\end{array}\right], \quad E_{+}=\left[\begin{array}{ll}
0 & 1 \\
0 & 0
\end{array}\right], \quad E_{-}=\left[\begin{array}{ll}
0 & 0 \\
1 & 0
\end{array}\right] .
$$

We also use the Hermite basis of the $\operatorname{sl}(2)$ algebra, $T^{i}=\frac{1}{2} \sigma^{i}(i=1,2,3)$, where $\sigma^{i}$ are Pauli matrices. Then we make use of the identity (5) to re-express the action (1) as

$$
S=-\frac{i}{2 \pi} \int_{X_{4}} \omega \wedge\left[\partial \varphi \wedge \bar{\partial} \varphi+\partial \chi \wedge \bar{\partial} \psi e^{-2 \varphi}\right] .
$$

In this paper, we assume that the space-time $X_{4}$ is flat; $h_{1 \overline{1}}=h_{2 \overline{2}}=\frac{1}{2}$ with the others zero, and use the notation $u=z^{1}, v=z^{2}\left(\bar{u}=\bar{z}^{1}, \bar{v}=\bar{z}^{2}\right)$. Then, the action reads

$$
S=\kappa \int d^{4} z\left(\partial_{\gamma} \varphi \partial_{\bar{\gamma}} \varphi+\partial_{\gamma} \chi \partial_{\bar{\gamma}} \psi e^{-2 \varphi}\right), \quad \kappa=-\frac{f_{\pi}^{2}}{8 \pi} .
$$

The energy-momentum tensor is given by

$$
\begin{aligned}
& T_{\alpha \beta}=\frac{\kappa}{2}\left(\partial_{\alpha} \varphi \partial_{\beta} \varphi+\partial_{\alpha} \psi \partial_{\beta} \chi e^{-2 \varphi}\right), \quad T_{\bar{\alpha} \bar{\beta}}=\frac{\kappa}{2}\left(\partial_{\bar{\alpha}} \varphi \partial_{\bar{\beta}} \varphi+\partial_{\bar{\alpha}} \chi \partial_{\bar{\beta}} \psi e^{-2 \varphi}\right), \\
& T_{\alpha \bar{\beta}}=T_{\bar{\beta} \alpha}=\frac{\kappa}{2}\left(\delta_{\alpha}^{\gamma} \delta_{\bar{\beta}}^{\bar{\delta}}-h_{\alpha \bar{\beta}} h^{\gamma \bar{\delta}}\right)\left(\partial_{\gamma} \varphi \partial_{\bar{\delta}} \varphi+\partial_{\gamma} \chi \partial_{\bar{\delta}} \psi e^{-2 \varphi}\right),
\end{aligned}
$$

with their conservation laws,

$$
\partial_{\beta} T_{\alpha \bar{\beta}}+\partial_{\bar{\beta}} T_{\alpha \beta}=0, \quad \partial_{\beta} T_{\bar{\alpha} \bar{\beta}}+\partial_{\bar{\beta}} T_{\bar{\alpha} \beta}=0 .
$$

The components of the current $J=J^{0} H+J^{-} E_{+}+J^{+} E_{-}$are given in terms of $\varphi(x)$, $\chi(x)$ and $\psi(x)$,

$$
\begin{aligned}
J^{0} & =\frac{i}{\pi} \omega \wedge\left(\partial \varphi+\psi \partial \chi e^{-2 \varphi}\right), \\
J^{+} & =\frac{i}{\pi} \omega \wedge\left(\partial \psi-2 \partial \varphi \psi-\psi^{2} \partial \chi e^{-2 \varphi}\right), \quad J^{-}=\frac{i}{\pi} \omega \wedge \partial \chi e^{-2 \varphi},
\end{aligned}
$$


whose $u, v$ components are defined from the conservation laws, $\bar{\partial} J^{a}=h^{\alpha \bar{\beta}} \partial_{\bar{\beta}} J_{\alpha}^{a} d^{4} z=0$, $(a=0,+,-)$,

$$
J_{\alpha}^{0}=\kappa\left(\partial_{\alpha} \varphi+\psi \partial_{\alpha} \chi e^{-2 \varphi}\right), J_{\alpha}^{+}=\kappa\left(\partial_{\alpha} \psi-2 \partial_{\alpha} \varphi \psi-\psi^{2} \partial_{\alpha} \chi e^{-2 \varphi}\right), \quad J_{\alpha}^{-}=\kappa \partial_{\alpha} \chi e^{-2 \varphi}
$$

The currents $J^{a}$ correspond to the holomorphic right-action symmetry with parameters $\epsilon_{a}\left(z^{1}, z^{2}\right) ; \delta_{R} g(x)=\epsilon_{0} g H+\epsilon_{+} g E_{+}+\epsilon_{-} g E_{-}$. Accordingly $\varphi(x), \chi(x)$ and $\psi(x)$ transform in the following way,

$$
\delta \varphi=\epsilon_{0}-\epsilon_{+} \psi, \quad \delta \psi=2 \epsilon_{0} \psi-\epsilon_{+} \psi^{2}+\epsilon_{-}, \quad \delta \chi=\epsilon_{+} e^{2 \varphi}
$$

Similarly, the components of the current $\bar{J}$ are given by

$$
\begin{aligned}
& \bar{J}^{0}=-\frac{i}{\pi} \omega \wedge\left(\bar{\partial} \varphi+\chi \bar{\partial} \psi e^{-2 \varphi}\right) \\
& \bar{J}^{+}=-\frac{i}{\pi} \omega \wedge \bar{\partial} \psi e^{-2 \varphi}, \quad \bar{J}^{-}=-\frac{i}{\pi} \omega \wedge\left(\bar{\partial} \chi-2 \bar{\partial} \varphi \chi-\chi^{2} \bar{\partial} \psi e^{-2 \varphi}\right) .
\end{aligned}
$$

The $\bar{u}, \bar{v}$ components of the $\bar{J}^{a}$ are

$$
\bar{J}_{\bar{\beta}}^{0}=\kappa\left(\partial_{\bar{\beta}} \varphi+\chi \partial_{\bar{\beta}} \psi e^{-2 \varphi}\right), \bar{J}_{\bar{\beta}}^{+}=\kappa \partial_{\bar{\beta}} \psi e^{-2 \varphi}, \bar{J}_{\bar{\beta}}^{-}=\kappa\left(\partial_{\bar{\beta}} \chi-2 \partial_{\bar{\beta}} \varphi \chi-\chi^{2} \partial_{\bar{\beta}} \psi e^{-2 \varphi}\right)
$$

The currents $\bar{J}^{a}$ correspond to the anti-holomorphic left-action symmetry with parameters $\bar{\epsilon}_{a}\left(\bar{z}^{1}, \bar{z}^{2}\right) ; \delta_{L} g(x)=\bar{\epsilon}_{0} H g+\bar{\epsilon}_{+} E_{+} g+\bar{\epsilon}_{-} E_{-} g$. Accordingly $\varphi(x), \chi(x)$ and $\psi(x)$ transform as

$$
\delta \varphi=\bar{\epsilon}_{0}-\bar{\epsilon}_{-} \chi, \quad \delta \psi=\bar{\epsilon}_{-} e^{2 \varphi}, \quad \delta \chi=2 \bar{\epsilon}_{0} \chi+\bar{\epsilon}_{+}-\bar{\epsilon}_{-} \chi^{2}
$$

\section{Two-toroidal Lie algebra}

To obtain a current algebra in the KWZW model, we start with the classical Poisson bracket (P.B.) in the light-cone frame. We take $\bar{u}$ as our time coordinate, while the space variables are denoted as $\mathbf{x}=(u, v, \bar{v})$. In this light-cone frame, the action (11) or (10) is first order in time derivatives, that is, it is already in Hamiltonian form. Therefore, we can define the P.B. without introducing conjugate momenta of fields $\phi^{i}=(\varphi, \psi, \chi)$. The 
formula applied to the 2D WZW model [11] can be easily extended to the present case as follows,

$$
\left\{X(\mathbf{x}), Y\left(\mathbf{x}^{\prime}\right)\right\}=\int d^{3} \tilde{\mathbf{x}} d^{3} \tilde{\mathbf{x}}^{\prime} \partial X(\mathbf{x}) / \partial \phi^{i}(\tilde{\mathbf{x}}) F^{i j}\left(\tilde{\mathbf{x}}, \tilde{\mathbf{x}}^{\prime}\right) \partial Y\left(\mathbf{x}^{\prime}\right) / \partial \phi^{j}\left(\tilde{\mathbf{x}}^{\prime}\right)
$$

where $F^{i j}$ is the inverse of the matrix $F_{i j}$ defined from the variation of the action, $\delta S=$ $\int d \bar{u} \int d^{3} \mathbf{x} d^{3} \mathbf{x}^{\prime} F_{i j}\left(\mathbf{x}, \mathbf{x}^{\prime}\right) \delta \phi^{i}(\mathbf{x}) \partial_{\bar{u}} \phi^{j}\left(\mathbf{x}^{\prime}\right)$

$$
F^{i j}\left(\mathbf{x}, \mathbf{x}^{\prime}\right)=\frac{1}{2 \kappa}\left[\begin{array}{ccc}
-\frac{1}{2} & 0 & \chi(\mathbf{x})-\chi\left(\mathbf{x}^{\prime}\right) \\
0 & 0 & -e^{2 \varphi(\mathbf{x})} \\
-\left(\chi(\mathbf{x})-\chi\left(\mathbf{x}^{\prime}\right)\right) & -e^{2 \varphi\left(\mathbf{x}^{\prime}\right)} & \left(\chi(\mathbf{x})-\chi\left(\mathbf{x}^{\prime}\right)\right)^{2}
\end{array}\right] \epsilon\left(u-u^{\prime}\right) \delta^{2}\left(v-v^{\prime}\right)
$$

The function $\epsilon\left(u-u^{\prime}\right)$ is the sign function, and $\delta^{2}\left(v-v^{\prime}\right)=\delta\left(v-v^{\prime}\right) \delta\left(\bar{v}-\bar{v}^{\prime}\right)$. Henceforth, we take the values of the coordinates real by using an analytic continuation.

Since $\bar{u}$ is our time coordinate, from the conservation laws, $J_{u}^{i}$ are identified with the generators of the $J$-transformation (15), while $T_{u u}, T_{v u}, T_{\bar{v} u}$ the generators for space translations and $T_{\bar{u} u}$ the Hamiltonian density. If we take the $u$ as the time coordinate instead and define the P.B. with respect to $u$, then $\bar{J}_{\bar{u}}^{i}$ become the generators of the $\bar{J}$-transformation (18).

In the following, we present various P.B.'s involving the currents $J_{u}^{i}$. Using (19), we have fundamental P.B.'s for the components of the group element $g(\mathbf{x})$,

$$
\left\{g_{i j}(\mathbf{x}), g_{k l}\left(\mathbf{x}^{\prime}\right)\right\}=\frac{1}{4 \kappa}\left(g_{i j}(\mathbf{x}) g_{k l}\left(\mathbf{x}^{\prime}\right)-2 g_{k j}(\mathbf{x}) g_{i l}\left(\mathbf{x}^{\prime}\right)\right) \epsilon\left(u-u^{\prime}\right) \delta^{2}\left(v-v^{\prime}\right) .
$$

P.B.'s of $g(x)$ and $J_{u}^{a}$ are given in the matrix form as

$$
\begin{aligned}
& \left\{g(\mathbf{x}), J_{u}^{-}\left(\mathbf{x}^{\prime}\right)\right\}=g(\mathbf{x}) E_{-} \delta^{3}\left(\mathbf{x}-\mathbf{x}^{\prime}\right), \quad\left\{g(\mathbf{x}), J_{u}^{+}\left(\mathbf{x}^{\prime}\right)\right\}=g(\mathbf{x}) E_{+} \delta^{3}\left(\mathbf{x}-\mathbf{x}^{\prime}\right) \\
& \left\{g(\mathbf{x}), J_{u}^{0}\left(\mathbf{x}^{\prime}\right)\right\}=\frac{1}{2} g(\mathbf{x}) H \delta^{3}\left(\mathbf{x}-\mathbf{x}^{\prime}\right)
\end{aligned}
$$

or, in the Hermite basis, $J^{3}=J^{0}, J^{1}=\frac{1}{2}\left(J^{+}+J^{-}\right), J^{2}=\frac{1}{2 i}\left(J^{+}-J^{-}\right)$, the above P.B.'s are expressed concisely as

$$
\left\{g(\mathbf{x}), J_{u}^{i}\left(\mathbf{x}^{\prime}\right)\right\}=g(\mathbf{x}) T^{i} \delta^{3}\left(\mathbf{x}-\mathbf{x}^{\prime}\right)
$$


From (22), we can easily see that $J_{u}^{a}$ generate the transformation (15). For later use, we introduce $G^{i j}=\frac{1}{2} \operatorname{Tr} T^{i} g^{-1} T^{j} g$, which satisfies the following identities: $G^{i l} G^{j k} \delta_{i j}=\delta_{l k}$, $G^{l i} G^{k j} \delta_{i j}=\delta_{l k}$. The P.B.'s of the $G^{i j}$ and the currents $J_{u}^{k}$ are

$$
\left\{G^{i j}(\mathbf{x}), J_{u}^{k}\left(\mathbf{x}^{\prime}\right)\right\}=-i \epsilon^{i k l} G^{l j}(\mathbf{x}) \delta^{3}\left(\mathbf{x}-\mathbf{x}^{\prime}\right)
$$

P.B.'s among $J_{u}^{i}$ 's are in the Hermite basis,

$$
\left\{J_{u}^{i}(\mathbf{x}), J_{u}^{j}\left(\mathbf{x}^{\prime}\right)\right\}=-i \epsilon^{i j k} J_{u}^{k}(\mathbf{x}) \delta^{3}\left(\mathbf{x}-\mathbf{x}^{\prime}\right)+\frac{\kappa}{2} \delta^{i j} \partial_{u} \delta^{3}\left(\mathbf{x}-\mathbf{x}^{\prime}\right)
$$

and those among $J_{v}^{i}$ and $J_{u}^{j}$ are

$$
\left\{J_{v}^{i}(\mathbf{x}), J_{u}^{j}\left(\mathbf{x}^{\prime}\right)\right\}=-i \epsilon^{i j k} J_{v}^{k}(\mathbf{x}) \delta^{3}\left(\mathbf{x}-\mathbf{x}^{\prime}\right)+\frac{\kappa}{2} \delta^{i j} \partial_{v} \delta^{3}\left(\mathbf{x}-\mathbf{x}^{\prime}\right)
$$

P.B.'s of $J_{u}^{i}$ with $\bar{J}_{\bar{v}}^{j}$ and $\bar{J}_{\bar{u}}^{j}$ do not vanish, unlike the case of the 2D WZW model;

$$
\begin{aligned}
& \left\{\bar{J}_{\bar{v}}^{j}(\mathbf{x}), J_{u}^{i}\left(\mathbf{x}^{\prime}\right)\right\}=-\frac{\kappa}{2} \partial_{\bar{v}^{\prime}}\left(G^{i j}\left(\mathbf{x}^{\prime}\right) \delta^{3}\left(\mathbf{x}-\mathbf{x}^{\prime}\right)\right) \\
& \left\{\bar{J}_{\bar{u}}^{j}(\mathbf{x}), J_{u}^{i}\left(\mathbf{x}^{\prime}\right)\right\}=\frac{\kappa}{4} \partial_{\bar{v}^{\prime}}\left(G^{i j}\left(\mathbf{x}^{\prime}\right) \epsilon\left(u-u^{\prime}\right) \partial_{v} \delta^{2}\left(v-v^{\prime}\right)\right)
\end{aligned}
$$

Note, however, that right-hand sides of (27) and (28) are total derivatives in the coordinate $\bar{v}^{\prime}$ and hence can be dropped when we integrate the currents $J_{u}^{i}\left(\mathbf{x}^{\prime}\right)$ with respect to $\bar{v}^{\prime}$.

To define our current algebra properly, we compactify space directions such that $u, v$ and $\bar{v}$ take the values $I=[0,2 \pi]$. The charge of the $J$-transformation (15) is then given by

$$
Q^{i}=\int_{0}^{2 \pi} d u d v \epsilon_{i}(u, v) \tilde{J}^{i}, \quad \tilde{J}^{i}=\int_{0}^{2 \pi} d \bar{v} J_{u}^{i}(\mathbf{x})
$$

From the conservation laws, we see that $\tilde{J}^{i}$ are functions of $u$ and $v$ only; $\tilde{J}^{i}=\tilde{J}^{i}(u, v)$. We use the notation $\mathbf{z}=(u, v)$. Then the P.B.'s for $\tilde{J}^{i}(\mathbf{z})$ are written as

$$
\left\{\tilde{J}^{i}(\mathbf{z}), \tilde{J}^{j}\left(\mathbf{z}^{\prime}\right)\right\}=-i \epsilon^{i j k} \tilde{J}^{k}(\mathbf{z}) \delta^{2}\left(\mathbf{z}-\mathbf{z}^{\prime}\right)+\pi \kappa \delta^{i j} \partial_{u} \delta^{2}\left(\mathbf{z}-\mathbf{z}^{\prime}\right)
$$


This is also noted in [2]. We list other P.B.'s involving $\tilde{J}^{i}(\mathbf{z})$,

$$
\begin{aligned}
& \left\{g(\mathbf{x}), \tilde{J}^{i}\left(\mathbf{z}^{\prime}\right)\right\}=g(\mathbf{x}) T^{i} \delta^{2}\left(\mathbf{z}-\mathbf{z}^{\prime}\right), \\
& \left\{G^{i j}(\mathbf{x}), \tilde{J}^{k}\left(\mathbf{z}^{\prime}\right)\right\}=-i \epsilon^{i k l} G^{l j}(\mathbf{x}) \delta^{2}\left(\mathbf{z}-\mathbf{z}^{\prime}\right), \\
& \left\{J_{v}^{i}(\mathbf{x}), \tilde{J}^{j}\left(\mathbf{z}^{\prime}\right)\right\}=-i \epsilon^{i j k} J_{v}^{k}(\mathbf{x}) \delta^{2}\left(\mathbf{z}-\mathbf{z}^{\prime}\right)+\frac{\kappa}{2} \delta^{i j} \partial_{v} \delta^{2}\left(\mathbf{z}-\mathbf{z}^{\prime}\right), \\
& \left\{\bar{J}_{\bar{v}}^{i}(\mathbf{x}), \tilde{J}^{j}\left(\mathbf{z}^{\prime}\right)\right\}=0, \quad\left\{\bar{J}_{\bar{u}}^{i}(\mathbf{x}), \tilde{J}^{j}\left(\mathbf{z}^{\prime}\right)\right\}=0 .
\end{aligned}
$$

The equations in (34) mean that the currents $\tilde{J}^{j}$ are decoupled from $\bar{J}_{\bar{u}}^{i}$ and $\bar{J}_{\bar{v}}^{i}$, which is reminiscent of the decoupling of $J_{z}$ and $\bar{J}_{\bar{z}}$ sectors in the 2D WZW model.

The parameter functions $\epsilon_{i}(\mathbf{z})$ are defined on the torus and can be expanded as

$$
\epsilon_{i}(\mathbf{z})=\sum_{\mathbf{n}} \epsilon_{i}^{\mathbf{n}} e^{i \mathbf{n} \cdot \mathbf{z}}
$$

where $\mathbf{n}=\left(n_{1}, n_{2}\right) \in \mathbf{Z}^{2}$ and $\mathbf{n} \cdot \mathbf{z}=n_{1} u+n_{2} v$. Correspondingly, the modes of the currents $\tilde{J}^{i}(\mathbf{z})$ are defined as

$$
Q^{i}=\sum_{\mathbf{n}} \epsilon_{i}^{\mathbf{n}} J_{\mathbf{n}}^{i}, \quad J_{\mathbf{n}}^{i}=\int d u d v e^{i \mathbf{n} \cdot \mathbf{z}} \tilde{J}^{i}(\mathbf{z})
$$

Then from (30) we obtain

$$
\left\{J_{\mathbf{n}}^{i}, J_{\mathbf{m}}^{j}\right\}=-i \epsilon^{i j k} J_{\mathbf{n}+\mathbf{m}}^{k}+i \lambda_{1} n_{1} \delta^{i j} \delta_{\mathbf{n},-\mathbf{m}}, \quad \lambda_{1}=\frac{\pi^{2} f_{\pi}^{2}}{2} .
$$

We now present the mathematical interpretation of the P.B. algebra (37) obeyed by $J_{\mathbf{n}}^{i}$. We first note that, by restricting $\mathbf{n}$ to the subset $\mathbf{n}=\left(n_{1}, 0\right)$, (37) is reduced to the familiar affine KM algebra $\hat{s l}(2)$. For general $\mathbf{n}$, (37) defines an infinite-dimensional (i.e. $\mathbf{n} \in \mathbf{Z}^{2}$ ) Lie algebra with central extension. A few mathematicians have recently begun the study of the representation theory of this class of algebra [12]. In their terminology, (37) is called the two-toroidal Lie algebra $s l(2)_{\text {tor }}$ because of the double Fourier mode expansion (35). It is interesting that two-toroidal Lie algebra is obtained from the 4D KWZW model in the same fashion as the affine KM (one-toroidal) algebra from the 2D WZW model. 
The root system of $\hat{s l}(2)$ is two-dimensional, being infinite in one direction. For comparison, we have tentatively chosen a root system for the $s l(2)_{t o r}$ in close analogy with $\hat{s l}(2)$. It is then three-dimensional, being infinite in two directions. The Cartan matrices of affine KM algebras have vanishing determinants, while those of hyperbolic KM algebras have negative determinants. It is yet to be studied whether one can assign Cartan matrices to general n-toroidal Lie algebras. For the $\operatorname{sl}(2)_{\text {tor }}$ root system we have chosen, the "Cartan matrix" $K$ is

$$
K=\left[\begin{array}{rrr}
2 & -2 & -2 \\
-2 & 2 & 2 \\
-2 & 2 & 2
\end{array}\right] .
$$

The matrix $K$ has the features not shared by finite-dimensional and affine Lie algebras: its determinant has a double zero, and one of its off-diagonal elements is +2 , which is shown by a dotted double line in the corresponding Dynkin diagram in Fig.1.

The representation content of affine KM algebras can be understood based on highestweight $(\mathrm{HW})$ representations. There is no obvious extension of $\mathrm{HW}$ representations to two-toroidal Lie algebras because of the two-dimensionality of the infinite directions of their root systems. This makes it difficult to find a criterion for good representations.

Let us focus on the central term in (37). There appears only one center proportional to $\lambda_{1} n_{1}$, whereas a more general central term should consist of two terms, $\lambda_{1} n_{1}+\lambda_{2} n_{2}$,

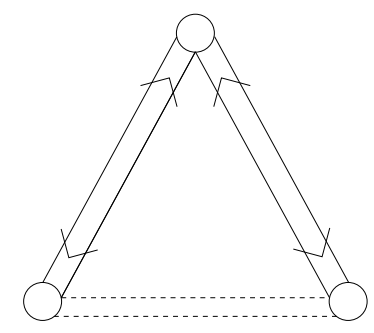

Figure 1: The Dynkin diagram for $s l(2)_{t o r}$. Lower two points are simple roots for two null directions while the upper point is the original $s l(2)$ simple root. 
by adding the new central charge $\lambda_{2}$. The $\lambda_{1}$ has to be quantized to ensure that the measure $\exp i S$ in the path-integral is well-defined [3], as in the 2D case. See also [2]. It is not clear how the quantization condition is related to the representation theory of the two-toroidal Lie algebra.

The two-toroidal Lie algebra is completed by constructing two kinds of derivations, $d_{1}$ and $d_{2}$, corresponding to $\left(n_{1}, n_{2}\right)$. We will see later that $d_{1}$ and $d_{2}$ are given by the components of the energy-momentum tensor $T_{u u}$ and $T_{v u}$, respectively, integrated over the space coordinates $\mathbf{x}$.

\section{Sugawara-like construction}

We now look into the possibility of the KWZW model being a good field theory realizing Sugawara's conjecture of the theory of currents [13]. From (11]), (14), (17), we obtain the following expression for the energy-momentum tensor,

$$
\begin{aligned}
& T_{\alpha \beta}=\frac{1}{2 \kappa}\left(J_{\alpha}^{0} J_{\beta}^{0}+\psi J_{\alpha}^{0} J_{\beta}^{-}-\psi J_{\alpha}^{-} J_{\beta}^{0}+J_{\alpha}^{+} J_{\beta}^{-}\right)=\frac{1}{2 \kappa} J_{\beta}^{i} J_{\alpha}^{j} M_{i j} \\
& T_{\bar{\alpha} \bar{\beta}}=\frac{1}{2 \kappa}\left(\bar{J}_{\bar{\alpha}}^{0} \bar{J}_{\bar{\beta}}^{0}+\chi \bar{J}_{\bar{\alpha}}^{0} \bar{J}_{\bar{\beta}}^{+}-\chi \bar{J}_{\bar{\alpha}}^{+} \bar{J}_{\bar{\beta}}^{0}+\bar{J}_{\bar{\beta}}^{+} \bar{J}_{\bar{\alpha}}^{-}\right)=\frac{1}{2 \kappa} \bar{J}_{\bar{\alpha}}^{i} \bar{J}_{\bar{\beta}}^{j} \bar{M}_{i j}
\end{aligned}
$$

where

$$
\begin{array}{rlrl}
M_{i j} & =\delta_{i j}+b_{i j} & & \bar{M}_{i j}={ }^{T} G^{i k}\left(\delta_{k l}+b_{k l}\right) G^{l j} \\
& =I+\left[\begin{array}{ccc}
0 & i & \psi \\
-i & 0 & -i \psi \\
-\psi & i \psi & 0
\end{array}\right], & =I+\left[\begin{array}{ccc}
0 & i & -\chi \\
-i & 0 & -i \chi \\
\chi & i \chi & 0
\end{array}\right] .
\end{array}
$$

$T_{\alpha \bar{\beta}}$ reads

$$
\begin{aligned}
T_{\alpha \bar{\beta}}=T_{\bar{\beta} \alpha} & =-\frac{1}{2 \kappa}\left(\delta_{\alpha}^{\gamma} \delta_{\bar{\beta}}^{\bar{\delta}}-h_{\alpha \bar{\beta}} h^{\gamma \bar{\delta}}\right)\left(J_{\gamma}^{0} \bar{J}_{\bar{\delta}}^{0}-\chi J_{\gamma}^{0} \bar{J}_{\bar{\delta}}^{+}-\psi J_{\gamma}^{-} \bar{J}_{\bar{\delta}}^{0}+J_{\gamma}^{-} \bar{J}_{\bar{\delta}}^{+}\left(\psi \chi+e^{2 \varphi}\right)\right) \\
& =-\frac{1}{2 \kappa}\left(\delta_{\alpha}^{\gamma} \delta_{\bar{\beta}}^{\bar{\delta}}-h_{\alpha \bar{\beta}} h^{\gamma \bar{\delta}}\right) J_{\gamma}^{i} \bar{J}_{\bar{\delta}}^{j} N_{i j}
\end{aligned}
$$


where

$$
N_{i j}=\left(\delta_{i k}+b_{i k}\right) G^{k j}=\left[\begin{array}{ccc}
\psi \chi+e^{2 \varphi} & i\left(\psi \chi+e^{2 \varphi}\right) & -\psi \\
-i\left(\psi \chi+e^{2 \varphi}\right) & \psi \chi+e^{2 \varphi} & i \psi \\
-\chi & -i \chi & 1
\end{array}\right] .
$$

Note that $M_{i j}, \bar{M}_{i j}$ and $N_{i j}$ contain extra terms which consist of $G^{i j}$ and $b_{i j}$. The term $b_{i j}$ corresponds to the torsion potential, whose curl is the torsion of the $S L(2, R)$ group manifold. This is different from the case of the 2D WZW model, where the torsion potential does not appear in the energy-momentum tensor. Thus, all components of the energy-momentum tensor are constructed from the set $\left(J_{\alpha}^{i}, \bar{J}_{\bar{\beta}}^{j}, G^{i j}, b_{i j}\right)$. Note that $T_{u u}$, $T_{v v}, T_{\bar{u} \bar{u}}$ and $T_{\bar{v} \bar{v}}$ are described by currents only since $b_{i j}$ is anti-symmetric.

P.B.'s of the currents $J_{u}^{k}$ and the generators for space translations, $T_{u u}, T_{v u}, T_{\bar{v} u}$ are given as

$$
\begin{aligned}
\left\{T_{u u}(\mathbf{x}), J_{u}^{k}\left(\mathbf{x}^{\prime}\right)\right\}= & \frac{1}{2} J_{u}^{k}(\mathbf{x}) \partial_{u} \delta^{3}\left(\mathbf{x}-\mathbf{x}^{\prime}\right), \\
\left\{T_{v u}(\mathbf{x}), J_{u}^{k}\left(\mathbf{x}^{\prime}\right)\right\}=\frac{1}{2} J_{u}^{k}(\mathbf{x}) \partial_{v} \delta^{3}\left(\mathbf{x}-\mathbf{x}^{\prime}\right) & +\frac{1}{4} \partial_{u}\left(\left(J_{v}^{k}(\mathbf{x})+b_{k j} J_{v}^{j}(\mathbf{x})\right) \delta^{3}\left(\mathbf{x}-\mathbf{x}^{\prime}\right)\right) \\
& -\frac{1}{4} \partial_{v}\left(\left(J_{u}^{k}(\mathbf{x})+b_{k j} J_{u}^{j}(\mathbf{x})\right) \delta^{3}\left(\mathbf{x}-\mathbf{x}^{\prime}\right)\right), \\
\left\{T_{\bar{v} u}(\mathbf{x}), J_{u}^{k}\left(\mathbf{x}^{\prime}\right)\right\}=\frac{1}{2} J_{u}^{k}(\mathbf{x}) \partial_{\bar{v}} \delta^{3}\left(\mathbf{x}-\mathbf{x}^{\prime}\right) & +\frac{1}{4} \partial_{u}\left(\left(J_{\bar{v}}^{k}(\mathbf{x})+b_{k j} J_{\bar{v}}^{j}(\mathbf{x})\right) \delta^{3}\left(\mathbf{x}-\mathbf{x}^{\prime}\right)\right) \\
& -\frac{1}{4} \partial_{\bar{v}}\left(\left(J_{u}^{k}(\mathbf{x})+b_{k j} J_{u}^{j}(\mathbf{x})\right) \delta^{3}\left(\mathbf{x}-\mathbf{x}^{\prime}\right)\right) .
\end{aligned}
$$

The P.B.'s of the $J_{u}^{k}$ and the Hamiltonian density $T_{\bar{u} u}$ are

$$
\begin{aligned}
\left\{T_{\bar{u} u}(\mathbf{x}), J_{u}^{k}\left(\mathbf{x}^{\prime}\right)\right\}=-\frac{1}{2} J_{v}^{k}(\mathbf{x}) \partial_{\bar{v}} \delta^{3}\left(\mathbf{x}-\mathbf{x}^{\prime}\right) & -\frac{1}{4} \partial_{v}\left(\left(J_{\bar{v}}^{k}+b_{k j} J_{\bar{v}}^{j}\right) \delta^{3}\left(\mathbf{x}-\mathbf{x}^{\prime}\right)\right) \\
& +\frac{1}{4} \partial_{\bar{v}}\left(\left(J_{v}^{k}+b_{k j} J_{v}^{j}\right) \delta^{3}\left(\mathbf{x}-\mathbf{x}^{\prime}\right)\right) .
\end{aligned}
$$

As promised above, $T_{u u}$ and $T_{v u}$ play the role of the derivations $d_{1}$ and $d_{2}$, respectively.

\section{Discussion}

An interesting physical application of the KWZW model is the computation of QCD multi-gluon amplitudes with all the same helicity, called maximally helicity violating 
(MHV) amplitudes [8]. This arises from the fact that the equation of motion of the KWZW model coincides with the SDYM equation. The latter equation has been pointed out to be related to a calculation technique of the MHV amplitudes [14. These amplitudes vanish at tree level and take a very simple form at one-loop. These simple results are believed to be due to the infinite-dimensional symmetry of the SDYM equation. Therefore, it is intriguing to reproduce these results from the viewpoint of the representation theory of two-toroidal current algebras. A relation between some MHV amplitudes and the $k=1 \mathrm{KM}$ algebra on $C P^{1}$ was already given by Nair [15].

It was suggested that the KWZW model can be obtained from an effective action of $4 \mathrm{D}$ chiral fermions coupled to the $G \times U(1)$ background gauge field [16]. We wish to point out that the field strength of the additional $U(1)$ factor, the Kähler potential, can be identified with the Kähler form $\omega$ in the KWZW model. In this case, the Kähler potential becomes dynamical since its kinematic term is also included in the effective action, which leads to the Plebanski equation for self-dual gravity [17]. The same action also appears as a space-time effective action in the $N=2$ string [7]. The quantum integrability of the string theory suggests that the current algebra and the classical integrability we have found in this paper survive in the quantum theory.

In this paper, we have constructed the two-toroidal Lie algebra in the KWZW model in the case of the $S L(2, R)$ group. The extension to the general $S L(N, R)$ case is straightforward. The fundamental P.B.'s for the group element $g(\mathbf{x}) \in S L(N, R)$ are

$$
\left\{g_{i j}(\mathbf{x}), g_{k l}\left(\mathbf{x}^{\prime}\right)\right\}=\frac{1}{2 N \kappa}\left(g_{i j}(\mathbf{x}) g_{k l}\left(\mathbf{x}^{\prime}\right)-N g_{k j}(\mathbf{x}) g_{i l}\left(\mathbf{x}^{\prime}\right)\right) \epsilon\left(u-u^{\prime}\right) \delta^{2}\left(v-v^{\prime}\right)
$$

and the $\operatorname{sl}(N)_{\text {tor }}$ algebra is derived from them. Note that the algebra (37) can be obtained also for cases when the Gauss decomposition is impossible, e.g. $G=S U(N)$. However, the treatment becomes more complicated in such cases. It appears that the two-toroidal algebra symmetry is responsible for the one-loop finiteness of the model. It is of interest to examine whether the same symmetry also renders the theory finite at all higher loops.

The NLSM in six dimensions is more badly ultra-violet divergent. One may add a Kähler Wess-Zumino term induced from the theory of 6D chiral fermions with the 
$G \times U(1)$ gauge field. It is interesting to see whether the NLSM with the additional term becomes one-loop finite and then whether a three-toroidal Lie algebra associated with an infinite-dimensional symmetry emerges.

We would like to thank Kiyoshi Higashijima, Minoru Wakimoto and Yoshihisa Saito for useful discussions. We are grateful to Patrick Dorey for a careful reading of the manuscript. This work is supported partially by Grant in Aid of the Ministry of Education, Science and Culture. T.U. is supported by the Japan Society for the Promotion of Science, No.6293. C.X. is supported by the COE (Center of Excellence) researchers program of the Ministry of Education, Science and Culture.

\section{References}

[1] A.M. Polyakov, Nucl. Phys. B164(1979)171.

J.L. Cardy, Nucl. Phys. B366(1991)403.

[2] V.P. Nair and J. Schiff, Phys. Lett. B246(1990)423; Nucl. Phys. B371(1992)329.

[3] A. Losev, G. Moore, N. Nekrasov and S. Shatashvili, Nucl. Phys. B46 (Proc. Suppl.) (1996)130.

[4] S. Donaldson, Proc. Lond. Math. Soc. 50(1985)1.

[5] K. Pohlmayer, Commun. Math. Phys. 72(1980)37.

[6] S.V. Ketov, Phys. Lett. B383(1996)390.

[7] H. Ooguri and C. Vafa, Nucl. Phys. B361(1991)469; Nucl. Phys. B367(1991)83. 
[8] G. Chalmers and W. Siegel, 'The self-dual sector of QCD amplitudes', hep-th/9606061.

D. Cangemi, 'Self-dual Yang-Mills Theory and One-Loop Like-Helicity QCD Multigluon Amplitudes', hep-th/9605208.

[9] C.N. Yang, Phys. Rev. Lett. 38(1977)1377.

[10] L-L. Chau, M-L. Ge and Y-S. Wu, Phys. Rev. D25(1982)1086.

L. Dolan, Phys. Lett. B113(1982)387.

L-L. Chau, Y-S. Wu, Phys. Rev. D26(1982)3581.

L-L. Chau, M-L. Ge, A. Sinha and Y-S. Wu, Phys. Lett. B121(1983)391.

[11] E. Witten, Commun. Math. Phys. 92(1984)455.

[12] R.V. Moody, S. Eswara Rao and T. Yokonuma, Geom. Dedicata 35(1990)283.

S. Eswara Rao and R.V. Moody, Commun. Math. Phys. 159(1994)239.

S. Berman and B. Cox, Pacific J. Math. 165(1994)239.

S. Berman and R.V. Moody, Invent. Math. 108(1992)323.

I.B. Frenkel and B.A. Khesin, Commun. Math. Phys. 178(1996)541.

[13] H. Sugawara, Phys. Rev. 170(1968)1659.

[14] W.A. Bardeen, Prog. Theor. Phys. Suppl. 123(1996)1.

[15] V.P. Nair, Phys. Lett. B214(1988)215.

[16] A. Losev, G. Moore, N. Nekrasov and S. Shatashvili, 'Chiral Lagrangians, Anomalies, Supersymmetry, and Holomorphy', hep-th/9606082.

[17] J.F. Plebanski, J. Math. Phys. 16(1975)2395. 